\title{
PRODUC'TS OF SPHERICAL DERIVATIVES AND NORMAL FUNCTIONS
}

\author{
CHEN HUAIHUI and PETER LAPPAN
}

(Received 23 June 1997; revised 4 September 1997)

\author{
Communicated by P. C. Fenton
}

\begin{abstract}
Chen and Gu [1] have given some results relating to normal families, and, in this paper, we give versions of these results valid for normal functions. In the process, we improve some of our previous results involving products of certain spherical derivatives as they relate to normal functions. Some examples are given to show the sharpness of our results.
\end{abstract}

1991 Mathematics subject classification (Amer. Math. Soc.): primary 30D05; secondary 30D35.

\section{Introduction}

Let $\mathbb{C}$ denote the complex plane. In [1], the first author and Y. Gu proved the following results about families of meromorphic functions.

THEOREM CG1. [1, Theorem 1, page 677] Let $k$ be a positive integer and let $F$ be a family of functions meromorphic on a domain $G \subset \mathbb{C}$, where each function in $F$ has only zeros of multiplicity at least $k$. If for each compact subset $K$ of $G$ there exist positive numbers $\delta, M, \alpha_{1}, \beta_{1}, \ldots, \alpha_{k-1}, \beta_{k-1}$ such that $\alpha_{j}+\beta_{j}=1$ for $1<j<k-1$ and $\left|f^{(k)}(z)\right|<M$ whenever both $f \in F$ and

$$
z \in K=\left\{w \in K: \sum_{j=1}^{k-1}\left|f^{(j)}(w)\right|+\sum_{j=1}^{k-1}\left|f^{(j)}(w)\right|^{\alpha_{j}}\left|f^{(k)}(w)\right|^{\beta_{j}}<\delta\right\},
$$

then $F$ is a normal family.

COROLLARY 1. [1, Corollary 2, page 677] Let $k$ be a positive integer and let $F$ be a family of functions meromorphic in a domain $G \subset \mathbb{C}$, where each function in $F$ has

(C) 1998 Australian Mathematical Society $0263-6115 / 98 \$ A 2.00+0.00$ 
only zeros of multiplicity at least $k$. If for each compact subset $K$ of $G$ there exist positive numbers $\alpha, \delta$, and $M$ such that $\left|f^{(k)}(z)\right| \leq M$ whenever $f \in F$ and

$$
z \in K_{f}=\left\{w \in K:|f(w)|^{\alpha}\left|f^{(k)}(w)\right| \leq \delta\right\},
$$

then $F$ is a normal family.

THEOREM CG2. [1, Theorem 2, page 677] Let $k$ be a positive integer and let $F$ be a family of functions meromorphic in a domain $G \subset \mathbb{C}$ such that each function in $F$ has only zeros of multiplicity at least $k$. If $F$ is not a normal family in some neighborhood of the point $z_{0} \in G$ then, for each positive number $\alpha<k$, there exists a sequence of points $\left\{z_{n}\right\}$ in $G$ such that $z_{n} \rightarrow z_{0}$, a sequence of positive numbers $\left\{\rho_{n}\right\}$ such that $\rho_{n} \rightarrow 0$, and a sequence of functions $\left\{f_{n}\right\}$ in $F$ such that, if $g_{n}(\zeta)=\left(\rho_{n}\right)^{-\alpha} f\left(z_{n}+\rho_{n} \zeta\right)$, then the sequence $\left\{g_{n}\right\}$ converges spherically and locally uniformly to a non-constant function meromorphic in the $\zeta$-plane.

Theorem CG2 is a generalization of results of Zalcman [5] and Pang [4].

Let $D=\{z:|z|<1\}$ denote the unit disk in the complex plane, and let Aut $(D)$ denote the collection of all conformal automorphisms of $D$ onto itself. A function $f$ meromorphic in $D$ is a normal function if the family $F=\{f \circ g: g \in \operatorname{Aut}(D)\}$ is a normal family on $D$. Since the results mentioned above are results about normal families and families which are not normal, it seems natural to expect that there would be corresponding results about normal functions and functions which are not normal functions. We show below that this is the case.

Let $f_{j}(z)$ denote the $\mathrm{j}$-th derivative of $f$, that is, $f_{j}(z)=f^{(j)}(z), j=0,1,2, \ldots$ We use

$$
f^{\#}(z)=\left|f^{\prime}(z)\right| /\left(1+|f(z)|^{2}\right)
$$

to denote the spherical derivative of $f$ at $z$. In [3], the second author proved the following results about normal functions and normal families.

THEOREM L1. If $f$ is a normal function in $D$ then for each integer $p \geq 1$ there exists a constant $M_{p}(f)$ such that

$$
\left(1-|z|^{2}\right)^{p} \prod_{j=1}^{p} f_{j-1}^{\#}(z) \leq M_{p}(f) .
$$

THEOREM L2. If $F$ is a normal family of functions meromorphic in a domain $G \subset \mathbb{C}$, then for each integer $p>1$ and each compact subset $K$ of $G$ there exists $a$ constant $M_{p}(K)$ such that

$$
\prod_{j=1}^{p} f_{j-1}^{\#}(z) \leq M_{p}(K, F)
$$


for each function $f \in F$ and $z \in K$.

A partial converse, for functions with no simple zeros, of Theorem L2 was proved by the authors in [2, Theorem 6$]$, as follows.

THEOREM CL. Let $k$ be a positive integer with $k \geq 3$, and let $F$ be a family of functions meromorphic in a domain $G \subset \mathbb{C}$ such that each function has only zeros of multiplicity at least $k$. If for each compact subset $K$ of $G$ there exists a positive number $M$ such that

$$
f_{k-1}^{\#}(z) f_{k-2}^{\#}(z) f_{k-3}^{\#}(z) \leq M
$$

for $f \in F$ and $z \in K$, then $F$ is a normal family. The conclusion remains true if we assume instead that $k \geq 2$ and we replace condition $(*)$ by the condition $f_{k-1}^{\#}(z) f_{k-2}^{\#}(z) \leq M$. The conclusion is also true if we assume only that $k \geq 1$ and we replace condition $(*)$ by the condition $f_{k-1}^{\#}(z) \leq M$.

In this paper we give a corresponding partial converse to Theorem L1.

The rest of the paper is organized as follows. Our Theorem 1, a version of Theorem CG1 which is valid for normal functions, is given in Section 2. In Section 3, we present a version of Theorem CG2 valid for normal functions, which is our Theorem 2. In Section 4, our Theorem 3 is a version of Theorem CL valid for normal functions. Finally, in Section 5, we give some examples relating to the sharpness of Theorem $\mathrm{Ll}$ and our Theorem 3.

\section{Sufficient conditions for a normal function}

LEMMA 1. Let $f(z)$ be a function meromorphic in D. If $\gamma \in \operatorname{Aut}(D)$ and $g(z)=$ $f(\gamma(z))$, then, for $k \geq 2$, we have

$$
\begin{aligned}
& \left|\left(1-|z|^{2}\right)^{k}\right| g^{(k)}(z)\left|-\left(1-|\gamma(z)|^{2}\right)\right| f^{(k)}(\gamma(z))|| \\
& \leq C_{k} \sum_{j=1}^{k-1}\left(1-|\gamma(z)|^{2}\right)\left|f^{(k)}(\gamma(z))\right|
\end{aligned}
$$

where $C_{k}$ is a constant depending only on $k$.

PROOF. Letting $\gamma(z)=e^{i \theta}\left(z-z_{0}\right) /\left(1-\overline{z_{0}} z\right)$, we have both

$$
\left|\gamma^{\prime}(z)\right|=\left(1-|\gamma(z)|^{2}\right) /\left(1-|z|^{2}\right)
$$

and

$$
\left|\gamma^{(j)}(z) / \gamma^{\prime}(z)\right| \leq 1 /(1-|z|)^{j-1} \quad \text { for } j \geq 2 \text {. }
$$


For $k \geq 2$, we have

$$
\begin{aligned}
g^{(k)}(z)= & f^{(k)}(\gamma(z))\left(\gamma^{\prime}(z)\right)^{k} \\
& +\sum_{j=1}^{k-1} \sum_{n_{1} \ldots, n_{k}} C_{k, j, n_{1}, \ldots, n_{k}} f^{(j)}(\gamma(z))\left(\gamma^{\prime}(z)\right)^{n_{k}} \cdots\left(\gamma^{(k)}(z)\right)^{n_{k}},
\end{aligned}
$$

where $n_{1}, n_{2}, \ldots, n_{k}$ satisfy the conditions $n_{1}+n_{2}+\cdots+n_{k}=j$ and $n_{1}+2 n_{2}+$ $\cdots+k n_{k}=k$ in each term of the sum. Thus,

$$
\begin{aligned}
\mid(1- & \left.|z|^{2}\right)^{k}\left|g^{(k)}(z)\right|-\left(1-|\gamma(z)|^{2}\right)^{k}\left|f^{(k)}(\gamma(z))\right| \mid \\
\leq & \left(1-|z|^{2}\right)^{k}\left|g^{(k)}(z)-f^{(k)}(\gamma(z))\left(\gamma^{\prime}(z)\right)^{k}\right| \\
\leq & \sum_{j=1}^{k-1} \sum_{n_{1}, \cdots, n_{k}} C_{k, j, n_{1}, \cdots, n_{k}}\left|f^{(j)}(\gamma(z))\right|\left|\gamma^{\prime}(z)\right|^{j} \\
& \times\left|\gamma^{\prime \prime}(z) / \gamma^{\prime}(z)\right|^{n_{2}} \cdots\left|\gamma^{(k)}(z) / \gamma^{\prime}(z)\right|^{n_{k}}\left(1-|z|^{2}\right)^{k} .
\end{aligned}
$$

Since

$$
\left|\gamma^{\prime}(z)\right|^{j}=\left(\left(1-|\gamma(z)|^{2}\right) /\left(1-\mid z^{2}\right)\right)^{j}
$$

and

$$
\begin{aligned}
\left|\gamma^{\prime \prime}(z) / \gamma^{\prime}(z)\right|^{n_{2}} \cdots\left|\gamma^{(k)}(z) / \gamma^{\prime}(z)\right|^{n_{k}} & \leq(1 /(1-|z|))^{n_{2}+2 n_{3}+\cdots+(k-1) n_{k}} \\
& =(1 /(1-|z|))^{k-j},
\end{aligned}
$$

we have

$$
\begin{aligned}
&\left|\left(1-|z|^{2}\right)^{k}\right| g^{(k)}(z)\left|-\left(1-|\gamma(z)|^{2}\right)^{k}\right| f^{(k)}(\gamma(z))|| \\
& \leq\left(1-|z|^{2}\right)^{k} \sum_{j=1}^{n} \sum_{n_{1} \ldots n_{k}} C_{k, j, n_{1} \ldots \ldots n_{k}}\left|f^{(j)}(\gamma(z))\right| \\
& \times\left(\left(1-|\gamma(z)|^{2}\right) /\left(1-|z|^{2}\right)\right)^{j}(1 /(1-|z|))^{k-j} \\
&= \sum_{j=1}^{k-1} \sum_{n_{1} \ldots . . n_{k}} C_{k, j, n_{1} \ldots, n_{k}}\left|f^{(j)}(\gamma(z))\right| \\
& \times\left(1-|z|^{2}\right)^{k-j}\left(1-|\gamma(z)|^{2}\right)^{j} \\
& \leq C_{k} \sum_{j=1}^{n}\left(1-|\gamma(z)|^{2}\right)^{j}\left|f^{(j)}(\gamma(z))\right| .
\end{aligned}
$$

This completes the proof of the lemma. 
The inequality (1) implies

$$
\begin{aligned}
& \left(1-|z|^{2}\right)^{k}\left|g^{(k)}(z)\right| \\
& \quad \leq\left(1-|\gamma(z)|^{2}\right)^{k}\left|f^{(k)}(\gamma(z))\right|+C_{k} \sum_{j=1}^{k-1}\left(1-|\gamma(z)|^{2}\right)^{j}\left|f^{(j)}(\gamma(z))\right| .
\end{aligned}
$$

If we replace $f, g, \gamma$, and $z$ by $g, f, \gamma^{-1}$, and $\gamma(z)$, respectively, (2) becomes

$$
\begin{aligned}
& \left(1-|\gamma(z)|^{2}\right)^{k}\left|f^{(k)}(\gamma(z))\right| \\
& \quad \leq\left(1-|z|^{2}\right)^{k}\left|g^{(k)}(z)\right|+C_{k} \sum_{j=1}^{k-1}\left(1-|z|^{2}\right)^{j}\left|g^{(j)}(z)\right| .
\end{aligned}
$$

By using the inequalities (2) and (3), we can modify Theorem CG1 into a result about a single function as follows.

THEOREM 1. Let $k$ be a positive integer and let $f$ be a function meromorphic in $D$ such that $f$ has only zeros of multiplicity at least $k$. If there exist positive numbers $\delta, M, \alpha_{1}, \beta_{1}, \ldots \alpha_{k-1}, \beta_{k-1}$ such that $\alpha_{j}+\beta_{j}=1$ for $1 \leq j \leq k-1$ and $\left(1-|z|^{2}\right)^{k}\left|f^{(k)}(z)\right| \leq M$ whenever

$z \in K=\left\{w \in D: \sum_{j=1}^{k-1}\left(1-|w|^{2}\right)^{j}\left|f^{(j)}(w)\right|+\sum_{j=1}^{k-1}\left(1-|w|^{2}\right)^{j \alpha_{j}+k \beta_{j}}\left|f^{(k)}(w)\right|^{\beta_{j}} \leq \delta\right\}$,

then $f$ is a normal function.

PROOF. It suffices to prove that the family $F=\{f(\gamma(z)): \gamma \in \operatorname{Aut}(D)\}$ is a normal family in $D$. First, let $g_{\gamma}(z)=f(\gamma(z)) \in F$. For $z \in D$, define $\phi_{\gamma}(z)$ by

$$
\phi_{\gamma}(z)=\sum_{j=1}^{k-1}\left(1-|z|^{2}\right)^{j}\left|g_{\gamma}^{(j)}(z)\right|+\sum_{j=1}^{k-1}\left(1-|z|^{2}\right)^{j \alpha+k \beta}\left|g_{\gamma}^{(j)}(z)\right|^{\alpha}\left|g^{(k)}(z)\right|^{\beta},
$$

where $\alpha+\beta=1$ and $\alpha / \beta=\min \left\{\alpha_{j} / \beta_{j}, 1 \leq j \leq k-1\right\}$. We claim that there exists a $\delta^{\prime}>0$ such that $\phi_{\gamma}(z) \leq \delta^{\prime}$ implies that $\gamma(z) \in K$. To show this, let $\left\{\gamma_{n}\right\}$ be a sequence in $\operatorname{Aut}(D)$ and let $\left\{z_{n}\right\}$ be a sequence of points in $D$ such that $\phi_{\gamma_{n}}\left(z_{n}\right) \rightarrow 0$, and let $g_{\gamma_{n}}(z)=f\left(\gamma_{n}(z)\right)$. Now $\phi_{\gamma_{n}}(z) \rightarrow 0$ means that

$$
\left(1-\left|z_{n}\right|^{2}\right)^{j}\left|g^{(j)}\left(z_{n}\right)\right| \rightarrow 0, \quad 1 \leq j \leq k-1,
$$

and

$$
\left(1-\left|z_{n}\right|^{2}\right)^{j \alpha+k \beta}\left|g_{\gamma_{n}}^{(j)}\left(z_{n}\right)\right|^{\alpha}\left|g_{\gamma_{n}}^{(k)}\left(z_{n}\right)\right|^{\beta} \rightarrow 0, \quad 1 \leq j \leq k-1 .
$$


By taking $\beta$-th roots, (5) becomes

$$
\left(1-\left|z_{n}\right|^{2}\right)^{(j \alpha+k \beta) / \beta}\left|g_{\gamma_{n}}\left(z_{n}\right)\right|^{\alpha / \beta}\left|g_{\gamma_{n}}^{(k)}\left(z_{n}\right)\right| \rightarrow 0, \quad 1 \leq j \leq k-1 .
$$

Now, using (3), we see that (4) implies

$$
\left(1-\left|\gamma_{n}\left(z_{n}\right)\right|^{2}\right)^{j}\left|f^{(j)}\left(\gamma\left(z_{n}\right)\right)\right| \rightarrow 0, \quad 1 \leq j \leq k-1,
$$

and using (3) again, we get

$$
\begin{aligned}
&\left(1-\left|\gamma_{n}\left(z_{n}\right)\right|^{2}\right)^{j \alpha_{j}+k \beta_{j}}\left|f^{(j)}\left(\gamma_{n}\left(z_{n}\right)\right)\right|^{\alpha_{j}}\left|f^{(k)}\left(\gamma_{n}\left(z_{n}\right)\right)\right|^{\beta_{j}} \\
& \leq\left(\left(1-\left|z_{n}\right|^{2}\right)^{j}\left|g_{\gamma_{n}}^{(j)}\left(z_{n}\right)\right|+C_{j} \sum_{p=1}^{j-1}\left(1-\left|z_{n}\right|^{2}\right)^{p}\left|g_{\gamma_{n}}^{(p)}\left(z_{n}\right)\right|^{\alpha_{j}}\right) \\
& \times\left(\left(1-\left|z_{n}\right|^{2}\right)^{k}\left|g_{\gamma_{n}}^{(k)}\left(z_{n}\right)\right|+C_{k} \sum_{p=1}^{k-1}\left(1-\left|z_{n}\right|^{2}\right)\left|g_{\gamma_{n}}^{(p)}\left(z_{n}\right)\right|\right)^{\beta_{j}} .
\end{aligned}
$$

Let

$$
\begin{aligned}
B_{n} & =\max _{0 \leq j \leq k-1}\left\{\left(1-\left|z_{n}\right|^{j}\right)\left|g_{\gamma_{n}}^{(j)}\left(z_{n}\right)\right|\right\} \\
& =\left(1-\left|z_{n}\right|^{2}\right)^{j_{n}}\left|g_{\gamma_{n}}^{\left(j_{n}\right)}\left(z_{n}\right)\right|,
\end{aligned}
$$

where $j_{n}$ is defined by this last expression. Then

$$
\begin{aligned}
(1- & \left.\left|\gamma_{n}\left(z_{n}\right)\right|^{2}\right)^{j \alpha_{j}+k \beta_{j}}\left|f^{(j)}\left(\gamma_{n}\left(z_{n}\right)\right)\right|^{\alpha_{j}}\left|f^{(k)}\left(\gamma_{n}\left(z_{n}\right)\right)\right|^{\beta_{j}} \\
& \leq k^{\alpha_{j}} C_{k}^{\alpha_{j}} B_{n}^{\alpha_{j}}\left(\left(1-\left|z_{n}\right|^{2}\right)^{k}\left|g_{\gamma_{n}}^{(k)}\left(z_{n}\right)\right|+k C_{k} B_{n}\right)^{\beta_{j}} \\
& =k^{\alpha_{j}} C_{k}^{\alpha_{j}}\left(\left(1-\left|z_{n}\right|^{2}\right)^{k}\left|g_{\gamma_{n}}^{(k)}\left(z_{n}\right)\right| B_{n}^{\alpha_{j} / \beta_{j}}+k C_{k} B n^{1+\alpha_{j} / \beta_{j}}\right)^{\beta_{j}} .
\end{aligned}
$$

From (4), we have $B_{n} \rightarrow 0$, and, from (6), we have

$$
\begin{aligned}
(1- & \left.\left|z_{n}\right|^{2}\right)^{k}\left|g_{\gamma_{n}}^{(k)}\left(z_{n}\right)\right| B_{n}^{\alpha_{j} / \beta_{j}} \\
& \leq\left(1-\left|z_{n}\right|^{2}\right)^{j_{n}}\left|g_{\gamma_{n}}^{\left(j_{n}\right)}\left(z_{n} \mid\right)^{\alpha / \beta}\left(1-\left|z_{n}\right|^{2}\right)^{k}\right| g_{\gamma_{n}}^{(k)}\left(z_{n}\right) \mid \\
& =\left(1-\left|z_{n}\right|^{2}\right)^{k+j_{n} \alpha / \beta}\left|g_{\gamma_{n}}^{\left(j_{n}\right)}\left(z_{n}\right)\right|^{\alpha / \beta}\left|g_{\gamma_{n}}^{(k)}\left(z_{n}\right)\right| \rightarrow 0 .
\end{aligned}
$$

Combining these inequalities, we have

(8) $\left(1-\left|\gamma_{n}\left(z_{n}\right)\right|^{2}\right)^{\alpha_{j}+k \beta_{j}}\left|f^{(j)}\left(\gamma_{n}\left(z_{n}\right)\right)\right|^{\alpha_{j}}\left|f^{(k)}\left(\gamma_{n}\left(z_{n}\right)\right)\right|^{\beta_{j}} \rightarrow 0, \quad 0 \leq j \leq k-1$.

It now follows from (7) and (8) that $\gamma_{n}\left(z_{n}\right) \in K$ for $n$ sufficiently large or, in other words, that there exists a $\delta^{\prime}>0$ such that $\phi_{\gamma}(z)<\delta^{\prime}$ implies that $\gamma(z) \in K$, which establishes our claim. 
Now let $\delta^{\prime}$ be the number described above, let $\gamma \in \operatorname{Aut}(D)$, let $g_{\gamma}=f \circ g$, and let $z \in D$ be such that $\phi_{\gamma}(z)<\delta^{\prime}$. Then $\gamma(z) \in K$ which means

$$
\sum_{j=1}^{k-1}\left(1-|z|^{2}\right)^{j}\left|f^{(j)}(\gamma(z))\right| \leq \delta .
$$

Also, since $\gamma(z) \in K$, we have $\left(1-|\gamma(z)|^{2}\right)^{k}\left|f^{(k)}(\gamma(z))\right| \leq M$. Thus, from (2), we have

$$
\left(1-|z|^{2}\right)^{k}\left|g^{(k)}(z)\right| \leq M+C_{k} \delta
$$

Let $0<r<1$ and $D_{r}=\{w:|w-z| \leq r\} \subset D$. Then, for $w \in D$ and $\phi_{\gamma}(w) \leq \delta^{\prime}$, we have

$$
\left|g_{\gamma}^{(k)}(w)\right| \leq\left(M+C_{k} \delta\right) /\left(1-r^{2}\right)^{k}=M^{\prime} .
$$

Thus, we can choose $r$ and apply Theorem CG1-using $D$ as the compact set, $F=$ $\{\gamma \in \operatorname{Aut}(D)\}$, and $M^{\prime}$ as the bound-to conclude that $F$ is locally a normal family on $D$. But this means that $F$ is a normal family on $D$, and consequently that $f$ is a normal function. This completes the proof.

The following is an immediate corollary of Theorem 1.

COROLlARY 2. Let $k$ be a positive integer and let $f$ be a function meromorphic in $D$, such that $f$ has only zeros of multiplicity at least $k$. If there exist positive numbers $\alpha, \delta$, and $M$ such that $\left(1-|z|^{2}\right)^{k}\left|f^{(k)}(z)\right| \leq M$ for

$$
z \in K=\left\{w \in D:\left(1-|w|^{2}\right)^{k}|f(w)|^{\alpha}\left|f^{(k)}(w)\right| \leq \delta\right\}
$$

then $f$ is a normal function.

\section{A behavior of non-normal functions}

We now prove a version of Theorem CG2 for normal functions.

THEOREM 2. Let $k$ be a positive integer and let $f$ be a function meromorphic in $D$ such that $f$ has only zeros of multiplicity at least $k$. If $f$ is not a normal function, then, for each positive number $\alpha<k$, there exist a sequence of points $\left\{z_{n}\right\}$ in $D$ such that $\left|z_{n}\right| \rightarrow 1$ and a sequence of positive numbers $\left\{\rho_{n}\right\}$ and such that $\rho_{n} /\left(1-|z|^{2}\right) \rightarrow 0$ for which the sequence

$$
\left\{g_{n}(\zeta)=\left(1-\left|z_{n}\right|^{2}\right)^{\alpha}\left(\rho_{n}\right)^{-\alpha} f\left(z_{n}+\rho_{n} \zeta\right)\right\}
$$

converges spherically and locally uniformly to a non-constant meromorphic function in the $\zeta$-plane. 
PROOF. If $f$ is not a normal function, then, by Corollary 2 , there exists a sequence of points $\left\{z_{n}^{\prime}\right\}$ such that both

$$
z_{n}^{\prime} \in K=\left\{w \in D:\left(1-|w|^{2}\right)^{k}|f(w)|^{\lambda}\left|f^{(k)}(w)\right| \leq 1\right\},
$$

where $\lambda=(k-\alpha) / \alpha$, and

$$
M_{n}^{\prime}=\left(1-\left|z_{n}^{\prime}\right|^{2}\right)^{k}\left|f^{(k)}\left(z_{n}^{\prime}\right)\right| \rightarrow \infty .
$$

For each $n$, there exists a positive number $r_{n}$, with $\left|z_{n}^{\prime}\right|<r_{n}<1$, such that

$$
\left(\left(r_{n}\right)^{2}-\left|z_{n}^{\prime}\right|^{2}\right)^{k}\left|f^{(k)}\left(z_{n}^{\prime}\right)\right| \geq M_{n}^{\prime} / 2 .
$$

If $D_{n}=\left\{z \in D:|z|<r_{n}\right\}$ and if $z_{n} \in D_{n} \cap K$ is such that

$$
M_{n}=\left(\left(r_{n}\right)^{2}-\left|z_{n}\right|^{2}\right)^{k}\left|f^{(k)}\left(z_{n}\right)\right|=\max _{z \in D_{n} \cap K}\left(\left(r_{n}\right)^{2}-|z|^{2}\right)\left|f^{(k)}(z)\right|,
$$

then $M_{n} \rightarrow \infty$. Define

$$
\rho_{n}=\left(\left(1-\left|z_{n}\right|^{2}\right)^{\alpha}\left|f^{(k)}(z)\right|\right)^{-1 /(k-\alpha)},
$$

and

$$
g_{n}(\zeta)=\left(1-\left|z_{n}\right|^{2}\right)^{\alpha}\left(\rho_{n}\right)^{-\alpha} f\left(z_{n}+\rho_{n} \zeta\right)
$$

Then

$$
\left|g^{(k)}(0)\right|=\left(1-\left|z_{n}\right|^{2}\right)^{\alpha}\left(\rho_{n}\right)^{k-\alpha}\left|f^{(k)}(z)\right|=1,
$$

and

$$
\left|g_{n}(\zeta)\right|^{\lambda}\left|g_{n}^{(k)}(\zeta)\right|=\left(1-\left|z_{n}\right|^{2}\right)^{k}\left|f\left(z_{n}+\rho_{n} \zeta\right)\right|^{\lambda}\left|f^{(k)}\left(z_{n}+\rho_{n} \zeta\right)\right|,
$$

so that

$$
\left|g_{n}(0)\right|^{\lambda}=\left|g_{n}(0)\right|^{\lambda}\left|g^{(k)}(0)\right|=\left(1-\left|z_{n}\right|^{2}\right)^{k}\left|f\left(z_{n}\right)\right|^{\lambda}\left|f^{(k)}\left(z_{n}\right)\right| \leq 1
$$

because $z_{n} \in K$. Thus, $\left|g_{n}(0)\right| \leq 1$ for each $n$. We note that

$$
\begin{aligned}
\rho_{n} & =\left(\left(1-\left|z_{n}\right|^{2}\right)^{\alpha}\left|f^{(k)}\left(z_{n}\right)\right|\right)^{-1 /(k-\alpha)} \\
& =\left(\left(1-\left|z_{n}\right|^{2}\right)^{\alpha} M_{n}\left(\left(r_{n}\right)^{2}-|z-n|^{2}\right)^{-k}\right)^{-1 /(k-\alpha)} \\
& \leq\left(1-\left|z_{n}\right|^{2}\right) M_{n}^{-1 /(k-\alpha)},
\end{aligned}
$$

which means that for $R>0$ and $n$ sufficiently large,

$$
\rho_{n} R<\left(1-\left|z_{n}\right|\right) M_{n}^{1 /(k-\alpha)} R<\left(1-\left|z_{n}\right|\right) / 2 .
$$


Thus, if $R$ is a fixed positive number, $|\zeta| \leq R$, and $n$ is sufficiently large, we have

$$
\begin{aligned}
& \left|g_{n}(\zeta)\right|^{\lambda}\left|g_{n}^{(k)}(\zeta)\right| \\
& \quad=\left\{\left(1-\left|z_{n}\right|^{2}\right) /\left(1-\left|z_{n}+\rho_{n} \zeta\right|^{2}\right)\right\}^{k}\left(1-\left|z+\rho_{n} \zeta\right|^{2}\right)^{k}\left|f\left(z_{n}+\rho_{n} \zeta\right)\right|^{\lambda}\left|f^{(k)}\left(z_{n}+\rho_{n} \zeta\right)\right| \\
& \quad \geq\left(\frac{2}{3}\right)^{k}\left(1-\left|z_{n}+\rho_{n} \zeta\right|^{2}\right)^{k}\left|f\left(z_{n}+\rho_{n} \zeta\right)\right|^{\lambda}\left|f^{(k)}\left(z_{n}+\rho_{n} \zeta\right)\right| .
\end{aligned}
$$

If $\left|g_{n}(\zeta)\right|^{\lambda}\left|g_{n}^{(k)}(\zeta)\right| \leq(2 / 3)^{k}$, then $z_{n}+\rho_{n} \zeta \in K$ by the definition of $K$, and

$$
\begin{aligned}
\left|g_{n}^{(k)}(\zeta)\right| & =\left|g_{n}^{(k)}(\zeta)\right| /\left|g_{n}^{(k)}(0)\right|=\left|f^{(k)}\left(z_{n}+\rho_{n} \zeta\right)\right| /\left|f^{(k)}\left(z_{n}\right)\right| \\
& \leq\left(\left(r_{n}^{2}-\left|z_{n}\right|^{2}\right) /\left(r_{n}^{2}-\left|z_{n}+\rho_{n} \zeta\right|^{2}\right)\right)^{k} \\
& \leq\left(\left(r_{n}+\left|z_{n}\right|\right) /\left(r_{n}+\left|z_{n}\right|-\left|\rho_{n} \zeta\right|\right)\right)^{k}\left(\left(r_{n}-\left|z_{n}\right|\right) /\left(r_{n}-\left|z_{n}\right|-\left|\rho_{n} \zeta\right|\right)\right)^{k} \\
& <4^{n}
\end{aligned}
$$

for $n$ sufficiently large. Thus, $\left\{g_{n}\right\}$ is a normal family in the $\zeta$-plane by Corollary 1 . By taking a subsequence, if necessary, we may assume that $\left\{g_{n}\right\}$ converges spherically and locally uniformly to a function $g$ in the $\zeta$-plane. The inequalities above imply that $|g(0)| \leq 1$ and $\left|g^{(k)}(0)\right|=1$. Thus, $g$ is a non-constant meromorphic function in the $\zeta$-plane, and the theorem is proved.

\section{Normal functions and products of spherical derivatives}

Theorem CL has a version valid for normal functions.

THEOREM 3. Let $f$ be a function meromorphic in $D$ such that all the zeros of $f$ are of multiplicity at least 3 . If there exist positive numbers $\delta$ and $M$ such that

$$
f^{\#}(z) f_{1}^{\#}(z) f_{2}^{\#}(z) \leq M
$$

whenever $z \in D$ is such that both $|f(z)| \leq \delta$ and $(1-|z|)\left|f^{\prime}(z)\right| \leq \delta$, then $f$ is a normal function. The conclusion remains true if all the zeros of $f$ are multiple zeros and condition (9) is replaced by either

$$
\left(1-|z|^{2}\right) f^{\#}(z) f_{1}^{\#}(z) \leq M \quad \text { or } \quad f_{1}^{\#}(z) \leq M
$$

whenever $z \in D$ is such that both $|f(z)| \leq \delta$ and $\left(1-|z|^{2}\right)\left|f^{\prime}(z)\right| \leq \delta$.

PROOF. We will give a proof only for the first statement in the theorem. The other statements can be obtained by obvious modifications of the proof of the first statement.

Suppose that (9) holds and that $f$ is not a normal function. If we let $\alpha$ be such that $1<\alpha<2$, then there exist sequences $\left\{z_{n}\right\}$ and $\left\{\rho_{n}\right\}$ which satisfy the conditions 
of Theorem 2, so that the sequence $\left\{g_{n}(\zeta)=\left(1-\left|z_{n}\right|^{2}\right)^{\alpha} \rho_{n}^{-\alpha} f\left(z_{n}+\rho_{n} z\right)\right\}$ converges spherically and uniformly on compact sets to a non-constant meromorphic function $\mathrm{g}$ on the $\zeta$-plane. Since each function $g$ has only zeros of multiplicity at least 3 , it follows that the limit function $g$ cannot be a polynomial of degree less than 3 . Hence, there exists a point $\zeta$ and a positive number $A$ such that $A^{-1} \leq\left|g^{(j)}(z)\right|<A$ for $0<j<3$. Thus, for $n$ sufficiently large, $(2 A)^{-1} \leq\left|g_{n}^{(j)}(z)\right| \leq 2 A$ for $0<j<3$. It follows that

$$
\begin{aligned}
(2 A)^{-3} & \leq\left|g_{n}^{(3)}(\zeta) g_{n}^{\prime \prime}(z) g_{n}^{\prime}(z)\right| \\
& \leq\left(\rho_{n} /\left(1-\left|z_{n}\right|^{2}\right)\right)^{-3 \alpha} \rho_{n}^{6} \prod_{j=1}^{3}\left|f^{(j)}\left(z_{n}+\rho_{n} \zeta\right)\right| \\
& =\left(\rho_{n} /\left(1-\left|z_{n}\right|^{2}\right)\right)^{-3 \alpha} \rho_{n}^{6} \prod_{j=0}^{2} f_{j}^{\#}\left(z_{n}+\rho_{n} \zeta\right) \prod_{j=0}^{2}\left(1+\mid f^{(j)}\left(z_{n}+\left.\rho_{n} \zeta\right|^{2}\right) .\right.
\end{aligned}
$$

Since $\alpha>1$, for $j=0,1,2$, we have

$$
\begin{aligned}
\left|f^{(j)}\left(z_{n}+\rho_{n} \zeta\right)\right| & =\left(\rho_{n} /\left(1-\left|z_{n}\right|^{2}\right)\right)^{\alpha} \rho_{n}^{-j}\left|g_{n}^{(j)}(\zeta)\right| \\
& \leq 2 A\left(\rho_{n} /\left(1-\left|z_{n}\right|^{2}\right)\right)^{\alpha-j}\left(1-\left|z_{n}\right|^{2}\right)^{-j}
\end{aligned}
$$

Thus, for $n$ sufficiently large, we have both $\left|f\left(z_{n}+\rho_{n} \zeta\right)\right| \leq \delta$ and $\left(1-\mid z_{n}+\right.$ $\left.\left.\rho_{n} \zeta\right|^{2}\right)\left|f^{\prime}\left(z_{n}+\rho_{n} \zeta\right)\right| \leq \delta$. By the assumptions given, we have $\prod_{j=0}^{2} f_{j}^{*}\left(z_{n}+\rho_{n} \zeta\right) \leq M$. Combining the above and letting $M^{\prime}=M\left(1+\delta^{2}\right)$, we get

$$
\begin{aligned}
(2 A)^{-3} \leq & \left|g_{n}^{(3)}(\zeta) g_{n}^{\prime \prime}(\zeta) g_{n}^{\prime}(\zeta)\right| \\
\leq & M\left(1+\delta^{2}\right)\left(\rho_{n} /\left(1-\left|z_{n}\right|^{2}\right)\right)^{-3 \alpha} \rho_{n}^{6} \times \prod_{j=1}^{2}\left(1+4 A^{2}\left(\rho_{n} /\left(1-\left|z_{n}\right|^{2}\right)\right)^{2 \alpha} \rho_{n}^{-2 j}\right) \\
\leq & M^{\prime} \rho_{n}^{6}\left(\rho_{n} /\left(1-\left|z_{n}\right|^{2}\right)\right)^{-3 \alpha}+4 M^{\prime}\left(\rho_{n} /\left(1-\left|z_{n}\right|^{2}\right)\right)^{-\alpha} \rho_{n}^{4} A^{2} \\
& +4 M^{\prime}\left(\rho_{n} /\left(1-\left|z_{n}\right|^{2}\right)\right)^{-\alpha} \rho_{n}^{4} A^{2}+16 M^{\prime} A^{4}\left(\rho_{n} /\left(1-\left|z_{n}\right|^{2}\right)\right)^{\alpha} \\
= & M^{\prime}\left(\rho_{n} /\left(1-\left|z_{n}\right|^{2}\right)\right)^{6-3 \alpha}\left(1-\left|z_{n}\right|^{2}\right)^{6}+4\left(\rho_{n} /\left(1-\left|z_{n}\right|^{2}\right)\right)^{2-\alpha} A^{2}\left(1-\left|z_{n}\right|^{2}\right)^{4} \\
& \left.+4 M\left(\rho_{n} /\left(1-\left|z_{n}\right|^{2}\right)\right)^{2-\alpha} A^{2}\left(1-\left|z_{n}\right|^{2}\right)^{2}+16\left(\rho_{n} /\left(1-\left|z_{n}\right|^{2}\right)\right)^{\alpha} A^{4}\right) \\
\rightarrow & 0
\end{aligned}
$$

since $\rho_{n} /\left(1-\left|z_{n}\right|^{2}\right) \rightarrow 0$. But $(2 A)^{-3}>0$, so we have arrived at a contradiction. This proves the first statement of the theorem. 


\section{Some examples}

By Theorem L1, if $f$ is a normal function then

$$
f^{\#}(z) f_{1}^{\#}(z) f_{2}^{\#}(z)<M_{p}(f) /\left(1-|z|^{2}\right)^{3} .
$$

In Theorem 3 above, we showed that if $f$ is meromorphic with all its zeros of order at least 3 , and if $f^{\#}(z) f_{1}^{\#}(z) f_{2}^{\#}(z)<M$ then $f$ is a normal function. This calls into question the sharpness of Theorem 3 and Theorem L1. We give some examples dealing with the sharpness of these results. The first example shows that Theorem L1 is sharp for $p=2$.

EXAMPLE 1. There exists a normal function $f$ such that $\left(1-|z|^{2}\right)^{2} f^{\#}(z) f_{1}^{\#}(z)$ is bounded, but $\left(1-|z|^{2}\right)^{2-\epsilon} f^{\#}(z) f_{1}^{\#}(z)$ is not bounded for any choice of $\epsilon>0$.

ProOF. Let $B(z)$ be a Blaschke product whose zeros $\left\{z_{n}\right\}$ are positive, real, and form an interpolating sequence, and let

$$
f(z)=z+\int_{0}^{z} B(z) /(1-w) d w .
$$

Then $f^{\prime}(z)=1+B(z) /(1-z)$ and $f^{\prime \prime}(z)=B^{\prime}(z) /(1-z)+B(z) /(1-z)^{2}$. Since

$$
\left(1-|z|^{2}\right)\left|f^{\prime}(z)\right| \leq 2(1-|z|)|1+B(z) /(1-z)| \leq 4,
$$

we have that $f$ is a Bloch function, and hence it is a normal function. Further,

$$
f^{\#}(z)=\left|f^{\prime}(z)\right| /\left(1+|f(z)|^{2}\right)=|1+B(z) /(1-z)|\left(1+|f(z)|^{2}\right)^{-1},
$$

and

$$
\left.f_{1}^{\#}(z)=\mid B^{\prime}(z) /(1-z)+B(z) /(1-z)^{2}\right) \mid\left\{1+|1+B(z) /(1-z)|^{2}\right\}^{-1} .
$$

Since $B(z)$ is a Blaschke product, and $\left\{z_{n}\right\}$ is an interpolating sequence, we have $B\left(z_{n}\right)=0$ and there exists a number $\delta>0$ such that $\left|B^{\prime}\left(z_{n}\right)\right| \geq \delta /\left(1-\mid z_{n}\right) \mid$ for each $n$. Thus, $f^{\#}\left(z_{n}\right)=1 /\left(1+\left|f\left(z_{n}\right)\right|^{2}\right)$ and

$$
f_{1}^{\#}\left(z_{n}\right)=\left|B^{\prime}\left(z_{n}\right)\right| /\left(2\left(1-z_{n}\right)\right) \geq \delta /\left(2\left(1-\left|z_{n}\right|\right)^{2}\right),
$$

so that $f^{\#}(z) f_{1}^{\#}(z)\left(1-\left|z_{n}\right|\right)^{2} \geq \delta / 2$. It follows that $f^{\#}\left(z_{n}\right) f_{1}^{\#}\left(z_{n}\right)(1-|z|)^{2-\epsilon} \rightarrow \infty$ for each $\epsilon>0$. However, since $f$ is a normal function, Theorem Ll says that $f^{\#}(z) f_{1}^{\#}(z)(1-|z|)^{2}$ is uniformly bounded. This proves the result. 
By a similar example, we can show that Theorem $\mathrm{Ll}$ is sharp for all $p \geq 3$.

EXAMPLE 2. Let $p$ be a positive integer, $p \geq 3$. There exists a normal function $f$ such that $\left(1-|z|^{2}\right)^{p} \prod_{j=1}^{p} f_{j-1}^{\#}(z)$ is bounded, but $\left(1-|z|^{2}\right)^{p-\epsilon} \prod_{j=1}^{p} f_{j-1}^{\#}(z)$ is not bounded for any $\epsilon>0$.

ProOF. Again, let $B(z)$ be a Blaschke product with zeros $\left\{z_{n}\right\}$ of order $p-1$ at each point of the interpolating sequence $\left\{z_{n}\right\}$, where the points $z_{n}$ are real and positive and

$$
f(z)=P(z)+\int_{0}^{z} B(w) /\left\{(1-w)(\ln (e / 1-w))^{2}\right\} d w,
$$

where

$$
P(z)=1+\sum_{j=1}^{p-1} z^{j} /\left(j ! 2^{j-1}\right)
$$

It is easy to verify that

$$
2^{-j} \leq\left|P^{(j)}(z)\right| \leq 2
$$

for each $j \geq 1$. Also, $f^{\prime}(z)=P^{\prime}(z)+B(z) /\left\{(1-z)(\ln (e /(1-z)))^{2}\right\}$ so $(1-|z|)\left|f^{\prime}(z)\right|$ is uniformly bounded in $D$, and so $f$ is a Bloch function and hence a normal function. Further, $B^{(j)}\left(z_{n}\right)=0$ for $0 \leq j \leq p-2$ since $z$ is a zero of order $p-1$, and since $B(z)$ is the $(p-1)$-th power of a Blaschke product $B_{*}(z)$ with simple zeros which form an interpolating sequence, we have $B(z)=\left(B_{*}(z)\right)^{p-1}$,

$$
B^{(p-1)}\left(z_{n}\right)=(p-1) !\left(\left(B_{*}\right)^{\prime}\left(z_{n}\right)\right)^{p-1},
$$

and $(1-|z|)\left|\left(B_{*}\right)^{\prime}(z)\right| \leq 1$ for $|z|<1$. So, as in Example 1, there exists a $\delta>0$ such that $\left(1-\left|z_{n}\right|\right)^{p-1}\left|B^{(p-1)}\left(z_{n}\right)\right| \geq \delta$. Using these results about derivatives of $B$ at the points $z_{n}$, we have

$$
2^{-j} \leq f^{(j)}\left(z_{n}\right)=\lambda_{n}<2 \text { for } 1 \leq j \leq p-1,
$$

and

$$
f^{(p)}\left(z_{n}\right)=B^{(p-1)}\left(z_{n}\right) /\left\{\left(1-z_{n}\right)\left(\ln \left(e /\left(1-z_{n}\right)\right)\right)^{2}\right\},
$$

so $\left(1-\left|z_{n}\right|\right)^{p}\left|f^{(p)}\left(z_{n}\right)\right| \rightarrow 0$, but $\left(1-\left|z_{n}\right|\right)^{p-\epsilon}\left|f^{(p)}\left(z_{n}\right)\right| \rightarrow \infty$ for each $\epsilon>0$. But $f^{(j)}\left(z_{n}\right), 0 \leq j \leq p$, is bounded and non-zero by construction (recall that $B^{(j)}\left(z_{n}\right)=0$ for $0 \leq j \leq p-1$ ) so $f_{j}^{\#}\left(z_{n}\right)$ is bounded and non-zero for $0 \leq j \leq p-1$. Thus, $\left(1-|z|^{2}\right)^{p-\epsilon} \prod_{j=1}^{p} f_{j-1}^{\#}(z)$ is unbounded for each $\epsilon>0$, but, by Theorem L1, since $f$ is a bounded function, and thus a normal function, $\left(1-|z|^{2}\right)^{p} \prod_{j=1}^{p} f_{j-1}^{\#}(z)$ is bounded. This completes the proof. 
In Examples 1 and 2 there is no attempt to control the multiplicity of the zeros, and thus these examples may not relate well to Theorem 3 . To address the sharpness of Theorem 3, we have following example in which the function $f(z)$ is a bounded function, but $f$ does not satisfy the sufficiency conditions for normality given in Theorem 3.

EXAMPLE 3. The function $f(z)=\exp ((z+1) /(z-1))$ is a bounded function for which $(1-|z|) f^{\#}(z) f_{1}^{\#}(z) f_{2}^{\#}(z)$ is unbounded in $D$ but $(1-|z|)^{1+\epsilon} f^{\#}(z) f_{1}^{\#}(z) f_{2}^{\#}(z)$ is bounded in $D$ for each $\epsilon>0$ and $f$ omits the value zero (and hence, for each positive integer $k, f$ has all its zeros of order at least $k$ ). Further, $\left(1-|z|^{2}\right) f^{\#}(z) f_{1}^{\#}(z)$ is unbounded but $\left(1-|z|^{2}\right)^{1+\epsilon} f^{\#}(z) f_{1}^{\#}(z)$ is bounded for each $\epsilon>0$.

ProOF. As $z \rightarrow 1$, we have $\left|f^{(j)}(z)\right| \approx 2^{j}|f(z)| /|z-1|^{2^{j}}$ for $j \geq 1$. Thus,

$$
\begin{aligned}
f^{\#}(z) f_{1}^{\#}(z) f_{2}^{\#}(z) \approx & \prod_{j=0}^{2}\left(2^{j+1}|z-1|^{-2(j+1)}|f(z)|\right) /\left(1+4^{j}|z-1|^{-4 j}|f(z)|^{2}\right) \\
= & \left(2|z-1|^{-2}|f(z)|\right) /\left(1+|f(z)|^{2}\right) \\
& \times\left(4|z-1|^{-4}|f(z)|\right) /\left(1+4|z-1|^{-4}|f(z)|^{2}\right) \\
& \times\left(8|z-1|^{-6}|f(z)|\right) /\left(1+16|z-1|^{-8}|f(z)|^{2}\right) \\
= & 64 /\left(|z-1|^{2}\right) \times 1 /\left(1+|f(z)|^{2}\right) \\
& \times|z-1|^{-2}|f(z)| /\left(1+4|z-1|^{-4}|f(z)|^{2}\right) \\
& \times|z-1|^{-8}|f(z)|^{2} /\left(1+16|z-1|^{-8}|f(z)|^{2}\right) .
\end{aligned}
$$

Denote this last product by $\phi(z)$. We note for future reference that each of the last three factors of $\phi(z)$ is less than 1 , so, as a crude estimate, $\phi(z) \leq 64 /|z-1|^{2}$ for all $z \in D$. For $\delta>0$ and $\delta$ sufficiently small, there exists a point $z_{\delta} \in D$ such that $\left|z_{\delta}-1\right|=\delta$ and $\left(1-\left|z_{\delta}\right|^{2}\right) /\left|z_{\delta}-1\right|^{2}=2 \ln 1 /\left|z_{\delta}-1\right|$, which means that $\left|f\left(z_{\delta}\right)\right|=\left|z_{\delta}-1\right|^{2}$. Then

$$
\begin{aligned}
\left(1-\left|z_{\delta}\right|^{2}\right) \phi\left(z_{\delta}\right)= & 128\left(\ln \left(1 /\left|z_{\delta}-1\right|\right)\right) \times 1 /\left(1+\left|z_{\delta}-1\right|^{4}\right) \\
& \times 1 / 5 \times\left|z_{\delta}-1\right|^{-4} /\left(1+16\left|z_{\delta}-1\right|^{-4}\right) \\
\approx & 8 / 5 \ln \left(1 /\left|z_{\delta}-1\right|\right) \rightarrow \infty \text { as } \delta \rightarrow 0 .
\end{aligned}
$$

Thus $\left(1-|z|^{2}\right) f^{\sharp}(z) f_{1}^{\#}(z) f_{2}^{\#}(z)$ is unbounded in $D$. However, the definition of $\phi(z)$ gives the estimate

$$
\phi(z) \leq 64|f(z)|^{3} /|z-1|^{12},
$$

so, if $|f(z)| \leq|z-1|^{4}$ then $\phi(z) \leq 64$ and

$$
\begin{aligned}
(1-|z|)^{1+\epsilon} f^{\#}(z) f_{1}^{\#}(z) f_{2}^{\#}(z) & \approx(1-|z|)^{1+\epsilon} \phi(z) \\
& \leq 64(1-|z|)^{1+\epsilon} \rightarrow 0 \text { as } z \rightarrow 1 .
\end{aligned}
$$


If we take $z$ such that $|f(z)|>|1-z|^{4}$, then we have

$$
|f(z)|=\exp \left\{\left(|z|^{2}-1\right) /|1-z|^{2}\right\}>|z-1|^{4},
$$

which implies

$$
\left(1-|z|^{2}\right) /|z-1|^{2}<4 \ln (1 /|z-1|) .
$$

We again use the estimate that $\phi(z) \leq 64 /|z-1|^{2}$ to obtain $\left(1-|z|^{2}\right)^{1+\epsilon} \phi(z) \leq$ $256\left(1-|z|^{2}\right)^{\epsilon} \ln (1 /|z-1|) \rightarrow 0$ as $z \rightarrow 1$ for each $\epsilon>0$. Thus, we have shown that $(1-|z|)^{1+\epsilon} f^{\#}(z) f_{1}^{\#}(z) f_{2}^{\#}(z)$ is bounded in $D$. (Here, $f(z)$ and all its derivatives are analytic for $z \neq 1$, so the only concern is with the behavior of $f(z)$ as $z \rightarrow 1$.)

For the second statement, we have that

$$
\begin{aligned}
\phi(z) & =f^{\#}(z) f_{1}^{\#}(z) \\
& \approx 8 /|z-1|^{2} \times 1 /\left(1+|f(z)|^{2}\right) \times|z-1|^{-4}|f(z)|^{2} /\left(1+4|z-1|^{-4}|f(z)|^{2}\right),
\end{aligned}
$$

and reasoning very similar to the above gives the desired results.

This example shows that the hypotheses of Theorem 3, in particular condition (9), cannot be relaxed very much, if at all.

We now show that the second statement in Theorem 3 is sharp.

EXAMPLE 4. Let $g(z)=\left(1 /(z-1)^{\beta}\right) \exp \{(z+1) /(z-1)\}, \beta>0$. Then $g$ is not a normal function, $g$ omits the value zero, and $(1-|z|) g^{\#}(z) g_{1}^{\#}(z)$ is unbounded but $(1-|z|)^{1+\epsilon} g^{\#}(z) g_{1}^{\#}(z)$ is bounded for each $\epsilon>0$.

ProOF. Let $f(z)=\exp \{(z+1) /(z-1)\}$ so that $g(z)=f(z) /(z-1)^{\beta}$. Then $\left|g^{(j)}(z)\right| \approx 2^{j}|z-1|^{-2 j+\beta}|f(z)|$, and we may define a function $\phi(z)$ by

$$
\begin{aligned}
g^{\#}(z) g_{1}^{\#}(z) \approx \phi(z)= & 2|z-1|^{-(2+\beta}|f(z)| /\left(1+|z-1|^{-2 \beta}|f(z)|\right) \\
& \times\left(4|z-1|^{-4-\beta}|f(z)|\right) /\left(1+4|z-1|^{-2 \beta-4}|f(z)|^{2}\right) .
\end{aligned}
$$

If we let $\left\{z_{n}\right\}$ be a sequence of points in $D$ satisfying both $\left|z_{n}-1\right|=1 / n$ and $\left(1-\left|z_{n}\right|^{2}\right) /\left|z_{n}-1\right|^{2}=\beta \ln \left(1 /\left|z_{n}-1\right|\right)$, then $\left|f\left(z_{n}\right)\right|=\left|z_{n}-1\right|^{\beta}$ so $\left|g\left(z_{n}\right)\right|=1$ and

$$
\left(1-\left|z_{n}\right|^{2}\right) g^{\#}(z) \approx \beta \ln \left(1 /\left|z_{n}-1\right|\right) \rightarrow \infty,
$$

so $g$ is not a normal function. Also, since both $\left|f\left(z_{n}\right)\right|=\left|z_{n}-1\right|^{\beta}$ and $\left(1-\left|z_{n}\right|^{2}\right) / \mid z_{n}-$ $\left.1\right|^{2}=\beta \ln \left(1 /\left|z_{n}-1\right|\right)$, we have

$$
\begin{aligned}
\left(1-\left|z_{n}\right|^{2}\right) g^{\#}(z) g_{1}^{\#}(z) \approx & 2\left(1-\left|z_{n}\right|^{2}\right) /\left|z_{n}-1\right|^{2} \times 1 /\left(1+\left|z_{n}-1\right|^{-2 \beta}\left|f\left(z_{n}\right)\right|^{2}\right) \\
& \times 4\left|z_{n}-1\right|^{-2 \beta-4}\left|f\left(z_{n}\right)\right|^{2} /\left(1+4\left|z_{n}-1\right|^{-2 \beta-4}\left|f\left(z_{n}\right)\right|^{2}\right) \\
= & 2 \beta \ln \left(1 /\left|z_{n}-1\right|\right) \times 1 / 2 \times\left(4\left|z_{n}-1\right|^{-4}\right) /\left(1+4\left|z_{n}-1\right|^{-4}\right) \\
\approx & \beta \ln \left(1 /\left|z_{n}-1\right|\right) \rightarrow \infty .
\end{aligned}
$$


This shows that $\left(1-|z|^{2}\right) g^{\#}(z) g_{1}^{\#}(z)$ is unbounded.

Now let $\epsilon>0$. If $|f(z)| \leq|z-1|^{\beta+3}$, then we have

$$
\phi(z)<8|z-1|^{-(6+2 \beta)}|f(z)|^{2} \leq 8 .
$$

If $|f(z)|>|z-1|^{\beta+3}$, then $\left(1-|z|^{2}\right) /|z-1|^{2} \leq(\beta+3) \ln (1 /|z-1|)$. Let $|f(z)|=$ $|z-1|^{\beta+3}|h(z)|$, where $|h(z)|>1$. Then, using the definition of $f(z)$ and ignoring the denominator in the first factor, we have the estimate

$$
\begin{aligned}
\phi(z) & \leq 2|z-1||h(z)| \times 4|z-1|^{-1}|h(z)| /\left(1+4|z-1|^{2}|h(z)|^{2}\right) \\
& =8|z-1|^{-2}|z-1|^{2}|h(z)|^{2} /\left(1+4|z-1|^{2}|h(z)|^{2}\right)<2|z-1|^{-2} .
\end{aligned}
$$

Hence,

$$
\begin{aligned}
\left(1-|z|^{2}\right)^{1+\epsilon} \phi(z) & \leq 2\left(1-|z|^{2}\right)^{\epsilon}\left(1-|z|^{2}\right) /|z-1|^{2} \\
& <2\left(1-|z|^{2}\right)^{\epsilon}(\beta+3) \ln (1 /|z-1|) \rightarrow 0
\end{aligned}
$$

as $|z| \rightarrow 1$. Thus, for $|z|<1$, we have $\left(1-|z|^{2}\right)^{1+\epsilon} g^{\#}(z) g_{1}^{\#}(z)$ is bounded for each choice of $\epsilon>0$.

Finally, we give an example showing that condition (9) of Theorem 3 cannot be relaxed by very much.

EXAMPLE 5. The non-normal function

$$
h(z)=(1-z)^{\beta} \exp \{(1+z) /(1-z)\}, \quad \beta>0
$$

satisfies the condition $\left(1-|z|^{2}\right)^{\epsilon} h^{\#}(z) h_{1}^{\#}(z) h_{2}^{\#}(z)$ is bounded for $\epsilon>\beta / 2$, and $h$ omits the value zero.

PROOF. Let $F(z)=\exp \{(1+z) /(1-z)\}$. Then $\left|h^{(j)}(z)\right| \approx 2^{j}|1-z|^{\beta-2 j}|F(z)|$ for $j \geq 0$. We note that $|F(z)| \geq 1$ for $z \in D$. Further, $h(z)$ is easily seen to have the two asymptotic values 0 and $\infty$ at $z=1$, so $h(z)$ is not a normal function. Then

$$
\begin{aligned}
h^{\#}(z) h_{1}^{\#}(z) h_{2}^{\#}(z) \approx & 2|1-z|^{\beta-2}|F(z)| /\left(1+|1-z|^{2 \beta}|F(z)|^{2}\right) \\
& \times 4|1-z|^{\beta-4}|F(z)| /\left(1+4|1-z|^{2 \beta-4}|F(z)|^{2}\right) \\
& \times 8|1-z|^{\beta-6}|F(z)| /\left(1+16|1-z|^{2 \beta-2}|F(z)|^{2}\right) \\
= & |1-z|^{-\beta}|F(z)|^{-1} /\left(1+|1-z|^{2 \beta}|F(z)|^{2}\right) \\
& \times 4|1-z|^{2 \beta-4}|F(z)|^{2} /\left(1+4|1-z|^{2 \beta-4}|F(z)|^{2}\right) \\
& \times 16|1-z|^{2 \beta-8}|F(z)|^{2} /\left(1+16|1-z|^{2 \beta-8}|F(z)|^{2}\right) \\
\leq & 1 /\left(|1-z|^{\beta}|F(z)|\right), \quad z \in D .
\end{aligned}
$$


If $|F(z)| \geq|1-z|^{-\beta}$, then $h^{\#}(z) h_{1}^{\#}(z) h_{2}^{\#}(z)$ is bounded. However, if $|F(z)|<|1-z|^{-\beta}$, then $\left(1-|z|^{2}\right) /|1-z|^{2}<\beta \ln (1 /|1-z|)$, which means that $\left\{\left(1-|z|^{2}\right) /|1-z|^{2}\right\}^{\beta / 2}<$ $(\beta \ln (1 /|1-z|))^{\beta / 2}$ and thus

$$
\left(1-|z|^{2}\right)^{\epsilon} h^{\#}(z) h_{1}^{\#}(z) h_{2}^{\#}(z) \approx\left(1-|z|^{2}\right)^{\epsilon-\beta / 2}(\beta \ln (1 /|1-z|))^{\beta / 2} /|F(z)| \rightarrow 0
$$

as $|z| \rightarrow 1$ whenever $\epsilon>\beta / 2$. Thus, in all cases, we have $\left(1-|z|^{2}\right)^{\epsilon} h^{\#}(z) h_{1}^{\#}(z) h_{2}^{\#}(z)$ is bounded in $\mathrm{D}$ for $\epsilon>\beta / 2$.

From Example 5, we see that the first statement of Theorem 3 is sharp, in the sense that the hypothesis

$$
f^{\#}(z) f_{1}^{\#}(z) f_{2}^{\#}(z) \text { is bounded }
$$

cannot be replaced by

$$
(1-|z|)^{\epsilon} f^{\#}(z) f_{1}^{\#}(z) f_{2}^{\#}(z) \text { is bounded for fixed } \epsilon>0 .
$$

(For a given $\epsilon>0$, we can take $\beta$ so small that $\epsilon>\beta / 2$ and use the construction in Example 5 to show that Theorem 3 is is not valid for that choice of $\epsilon$.)

\section{References}

[1] Chen Huaihui and Gu Yong-Xing, 'Improvement of Marty's criterion and its application', Science in China, Ser. A 36 (1993), 674-681.

[2] Chen Huaihui and P. Lappan, 'Normal families, orders of zeros, and omitted values', Ann. Acad. Sci. Fenn. Ser. A I Math. 21 (1996), 89-100.

[3] P. Lappan, 'The spherical derivative and normal functions', Ann. Acad. Sci. Fenn. Ser. A I Math. 3 (1977), 301-310.

[4] Pang Xue-Cheung, 'Bloch's principle and normal criterion', Science in China, Ser. A 32 (1989), $782-791$.

[5] L. Zalcman, 'A heuristic principle in complex function theory', Amer. Math. Monthly 82 (1975), 813-817.

Department of Mathematics

Nanjing Normal University

Nanjing, Jiangsu 210024

Peoples Republic of China

e-mail: hhchen@pine.njnu.edu.cn
Department of Mathematics

Michigan State University

East Lansing, Michigan 48824

USA

e-mail: plappan@math.msu.edu 